\title{
Ionization Potentials, Dissociation Energies and Statistical Fragmentation of Neutral and Positively Charged Small Carbon Clusters
}

\author{
S. Díaz-Tendero ${ }^{1}$, G. Sánchez ${ }^{1}$, P.-A. Hervieux ${ }^{2}$, M. Alcamí ${ }^{1}$, and F. Martín ${ }^{1}$ \\ ${ }^{1}$ Departamento de Química, C-9, Universidad Autónoma de Madrid, 28049 Madrid, Spain \\ ${ }^{2}$ Institut de Physique et Chimie des Matériaux de Strasbourg, \\ GONLO, 23 rue du Loess, 67034 Strasbourg, France
}

Received on 29 July, 2005

\begin{abstract}
Dissociation energies, ionization potentials and fragmentation dynamics of neutral, singly- and doubly charged small carbon clusters have been theoretically studied with a combination of the density functional theory, the coupled cluster method and the the statistical model microcanonical Metropolis Monte Carlo. The second ionization potential decreases with the cluster size and is larger than the first one, which also decreases with the size showing oscillations. Dissociation energies also oscillate with the cluster size, being those with an odd number of atoms more stable. $\mathrm{C}_{3}$ cluster has the largest dissociation energy. The combination of a statistical treatment for the cluster fragmentation with experimental results has allowed us to evaluate the energy distribution in collisions experiments.
\end{abstract}

Keywords: Carbon clusters; Fragmentation; Dissociation energy and ionization potential

\section{INTRODUCTION}

The decay of excited small carbon clusters has been experimentally widely studied [1-19]. In these experiments, the loss of neutral $\mathrm{C}_{3}$ has been found to be the dominant dissociation process for both charged and neutral clusters. Understanding the deexcitation processes observed in clusters collision experiments implies the knowledge of dissociation energies and ionization potentials of the evaporated fragments. The aim of this paper is to provide these values for small $\mathrm{C}_{n}^{q+}$ carbon clusters $(n=2-12$ and $q=0-2)$. A large number of theoretical studies focussing on the structural properties of these systems can be found in the literature (see the reviews [20, 21] and references therein), but there is no theoretical work that treats all the clusters at the same level of theory. In order to obtain the energetic properties presented in this work, we have consistently evaluated neutral, singly- and doubly-charged small carbon clusters at the same level of theory. In addition, a statistical treatment of the fragmentation process has been carried out. These simulations have allowed us to have a direct comparison with recent fragmentation experiments [19] and the evaluation of energetic properties as a function of the cluster charge and size. The paper is organized as follows. In section II, we briefly summarize the computational methods employed. Dissociation energies, ionization potentials and fragmentation properties of $\mathrm{C}_{n}^{q+}$ clusters are presented and discussed in section III. We summarize our results in section IV.

\section{COMPUTATIONAL DETAILS}

The statistical fragmentation has been carried out with the microcanonical Metropolis Monte Carlo (MMMC) method as described in reference [22]. In this method, one moves in phase space until a region with maximum statistical weight is found. A physical observable is then evaluated as a statistical average in this region of maximum probability. The statistical weight measures the number of physically accessible states at a fixed energy and is entirely determined by the microscopic properties of the fragments. These properties (geometries, harmonic frequencies, rotational constants and binding energies) have been evaluated with standard quantum chemistry calculations. In particular, we have applied the density functional theory (DFT) with the B3LYP functional for exchange and correlation. This functional combines the Becke's three parameter nonlocal hybrid exchange potential [23] with the nonlocal correlation functional of Lee, Yang and Parr [24]. The geometries have been optimized by using the $6-311+\mathrm{G}(3 \mathrm{df})$ basis set (B3LYP/6-311+G(3df)). The B3LYP functional has been proved to be a good choice for the description of carbon clusters [25]. In the case of small carbon clusters, the calculated geometries and the vibrational frequencies are very close to those obtained at higher levels of calculations [26-28]. More accurate values of electronic and binding energies have been obtained with the coupled cluster theory $\operatorname{CCSD}(\mathrm{T}) / 6-311+\mathrm{G}(3 \mathrm{df})$, which includes all single and double excitations, as well as triple excitations in a perturbative way [29], and made use of the B3LYP optimized geometry. The electronic energies obtained at this level of theory have been corrected with the zero point energy (ZPE) obtained from DFT vibrational analysis. All structure calculations have been performed with the Gaussian-98 program package [30].

\section{RESULTS}

We have evaluated several properties of carbon clusters with linear and cyclic geometries and with different spin multiplicities (singlet and triplet for the neutral and doubly charged species and doublet and quadruplet for the singly charged ones) [28]. To introduce all the isomers in the MMMC simulations has been shown to be crucial to correctly describe the fragmentation process [19, 22]. Dissociation energies and ionization potentials have been calculated taking 


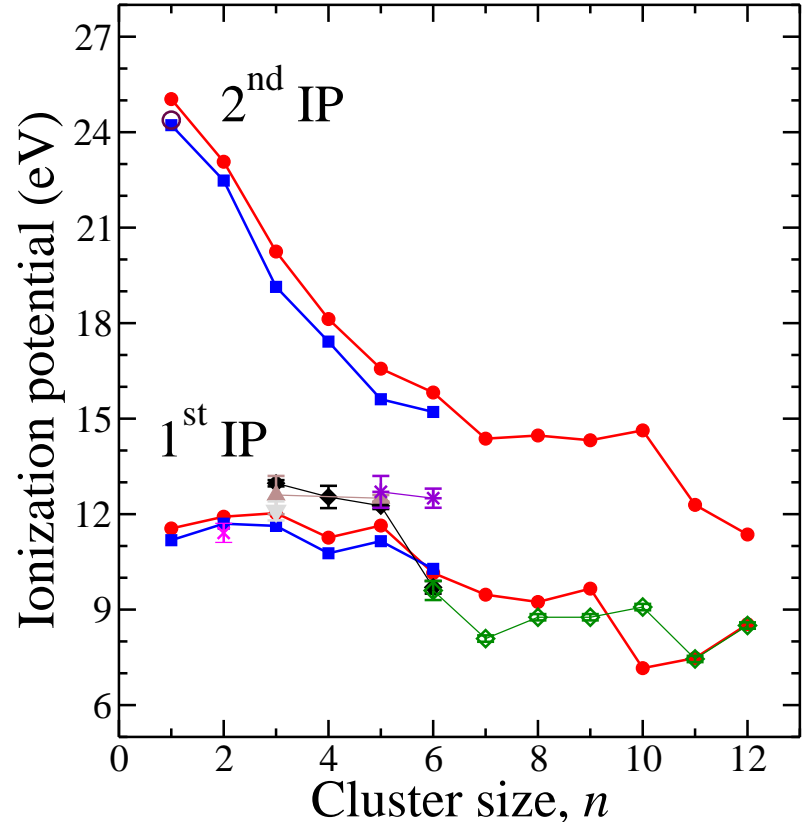

FIG. 1: First and second ionization potentials (in $\mathrm{eV}$ ) for $\mathrm{C}_{n}$ clusters as a function of the size. Circles: B3LYP/6-311+G(3df) level. Squares: CCSD(T)/6-311+G(3df)//B3LYP/6-311+G(3df) level. Experimental values are also included: Diamonds (Ref. [31]), up triangles (Ref. [32]), stars (Ref. [33]), open diamonds (Ref. [34]), crosses (Ref. [35]), down triangles (Ref. [36]), open squares (Ref. [37]), open circles (Ref. [38]).

the most stable isomer for each cluster size and charge, i.e., they are adiabatic energies.

\section{A. Ionization potentials}

We first present the results obtained for the adiabatic ionization potentials. Fig. 1 shows the first and second ionization potentials as a function of the cluster size evaluated at both B3LYP and CCSD(T) levels of theory. Differences between both methods in the first ionization potentials are never larger than $0.5 \mathrm{eV}$. Although for the second ionization potentials the differences are larger, they never exceed $1 \mathrm{eV}$. As a general trend, first IP slowly decreases with cluster size, but this decrease is not monotonic and present some oscillations. The first ionization potencial has been measured by different authors [31-39] and theoretically calculated by Giuffreda et al. [40]. We have also included in Fig. 1 the available experimental data. Our results agree reasonably well with the experimental measurements and those reported in ref. [40]. Second IP decreases with cluster size showing a stabilization for $n=8-10$. The observed behavior is quite predictable: the second IP is larger than the first IP and the difference between them is larger the smaller the system due to the ability of the larger cluster to accommodate multiple charges. Except for atomic $\mathrm{C}$, we are not aware of any experimental determination of the second ionization potential to compare with.

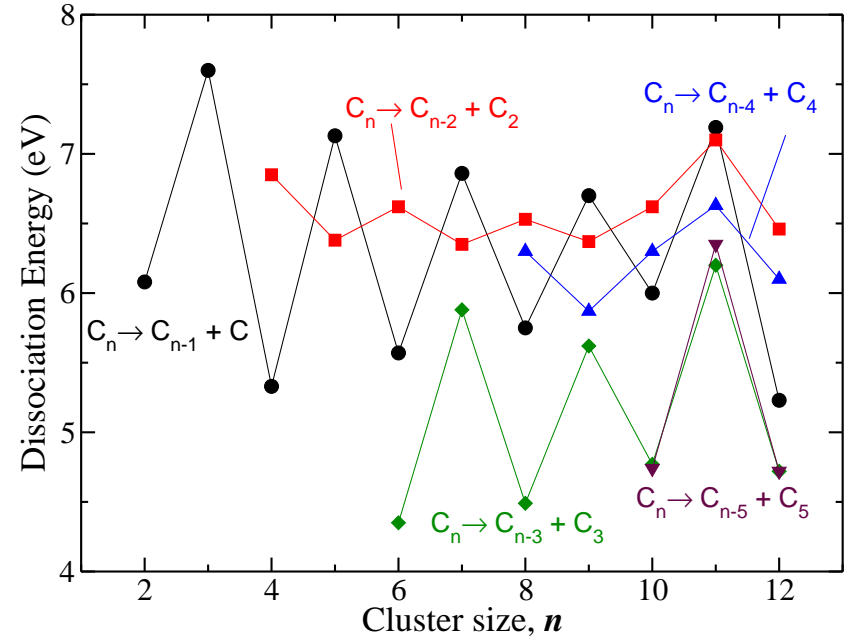

FIG. 2: Dissociation energies (in eV) of the process $\mathrm{C}_{n} \rightarrow \mathrm{C}_{n-x}+\mathrm{C}_{x}$ evaluated at the B3LYP/6-311+G(3df) level of theory. $x=1$ : circles, $x=2$ : squares, $x=3$ : diamonds, $x=4$ : triangles up, $x=5$ : triangles down.

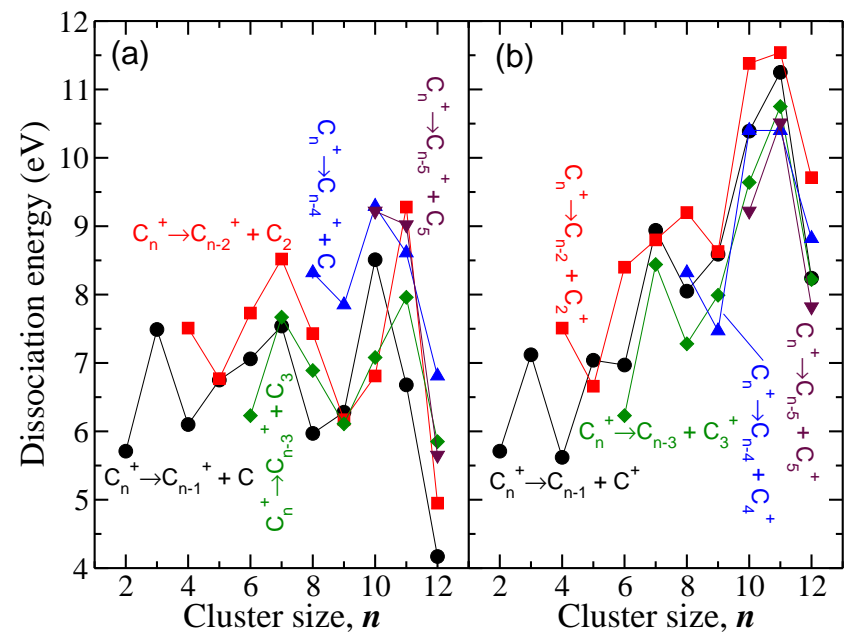

FIG. 3: Dissociation energies (in eV) of the processes (a) $\mathrm{C}_{n}^{+} \rightarrow$ $\mathrm{C}_{n-x}^{+}+\mathrm{C}_{x}$ and (b) $\mathrm{C}_{n}^{+} \rightarrow \mathrm{C}_{n-x}+\mathrm{C}_{x}^{+}$evaluated at the B3LYP/6$311+\mathrm{G}(3 \mathrm{df})$ level of theory. $x=1$ : circles, $x=2$ : squares, $x=3$ : diamonds, $x=4$ : triangles up, $x=5$ : triangles down.

\section{B. Dissociation energies}

The results for the dissociation energies of neutral, singlyand doubly-charged carbon clusters are presented in Figs. 2, 3 and 4 respectively. In these figures results of evaporation of $\mathrm{C}$ (circles), $\mathrm{C}_{2}$ (squares), $\mathrm{C}_{3}$ (diamonds), $\mathrm{C}_{4}$ (triangles up) and $\mathrm{C}_{5}$ (triangles down) are depicted.

In neutral clusters (Fig. 2) the lowest dissociation energy channels are $\mathrm{C}_{n-3} / \mathrm{C}_{3}$ and $\mathrm{C}_{n-5} / \mathrm{C}_{5}$. Alternation in dissociation energy is shown as a function of the cluster size. The oscillating behavior is more pronounced in the dissociation energy of channels corresponding to the evaporation of $\mathrm{C}$ and $\mathrm{C}_{3}$ and $\mathrm{C}_{5}$.

For singly charged $\mathrm{C}_{n}^{+}$clusters (Fig. 3) the lowest dissoci- 


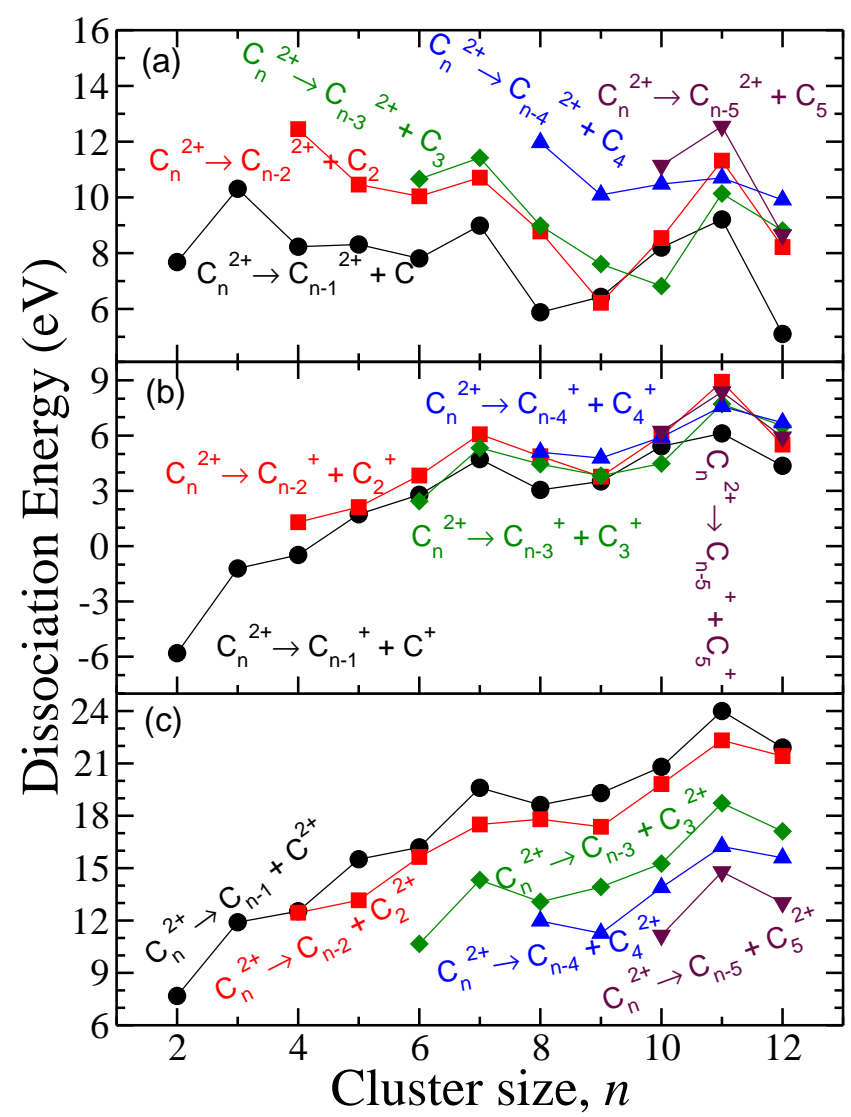

FIG. 4: Dissociation energies (in eV) of the processes (a) $\mathrm{C}_{n}^{2+} \rightarrow$ $\mathrm{C}_{n-x}^{2+}+\mathrm{C}_{x}$, (b) $\mathrm{C}_{n}^{2+} \rightarrow \mathrm{C}_{n-x}^{+}+\mathrm{C}_{x}^{+}$and (c) $\mathrm{C}_{n}^{2+} \rightarrow \mathrm{C}_{n-x}+\mathrm{C}_{x}^{2+}$ evaluated at the B3LYP/6-311+G(3df) level of theory. $x=1$ : circles, $x=2$ : squares, $x=3$ : diamonds, $x=4$ : triangles up, $x=5$ : triangles down.

ation energy channel is $\mathrm{C}_{n-1} / \mathrm{C}^{+}$for $n=2,3$ and $4 ; \mathrm{C}_{n-3}^{+} / \mathrm{C}_{3}$ for $n=6 ; \mathrm{C}_{n-2}^{+} / \mathrm{C}_{2}$ for $n=5$ and 10 and $\mathrm{C}_{n-1}^{+} / \mathrm{C}$ for $n=7,8,11$ and 12. In Fig. 3 alternation in the dissociation energy as a function of the cluster size is also shown but in this case it is less pronounced than for the neutral species.

For the smallest doubly charged carbon clusters $\left(\mathrm{C}_{n}^{2+}\right.$, with $n=2,3$ and 4) the dissociation energy of channel $\mathrm{C}_{n-1}^{+} / \mathrm{C}^{+}$is smaller than zero, i.e., they are metastable. These results are in agreement with calculations of the potential energy surface evaluated with multireference methods (MR-DCI) [41, 42]. The smallest doubly charged cluster that is stable against Coulomb explosion is $\mathrm{C}_{5}^{2+}[28,43]$. The lowest dissociation energy of this molecule corresponds to channel $\mathrm{C}_{4}^{+} / \mathrm{C}^{+}$. It is worth mentioning that in the experimental work of Chabot $e t$ al. [15] they found that this channel is the dominant one in the fragmentation of $\mathrm{C}_{5}^{2+}$. In addition, the dissociation energy of this channel is $\sim 1 \mathrm{eV}$ smaller than the required for the $\mathrm{C}_{3}^{+} / \mathrm{C}_{2}^{+}$ channel.

The lowest dissociation energy channel for $\mathrm{C}_{n}^{2+}$ corresponds to $\mathrm{C}_{n-1}^{+} / \mathrm{C}^{+}$, except for $\mathrm{C}_{6}^{2+}$ and $\mathrm{C}_{10}^{2+}$, closely followed by $\mathrm{C}_{n-3}^{+} / \mathrm{C}_{3}^{+}$, which is the energetically most favorable for $\mathrm{C}_{6}^{2+}$ and $\mathrm{C}_{10}^{2+}$. As a general trend, the channels in which the charge

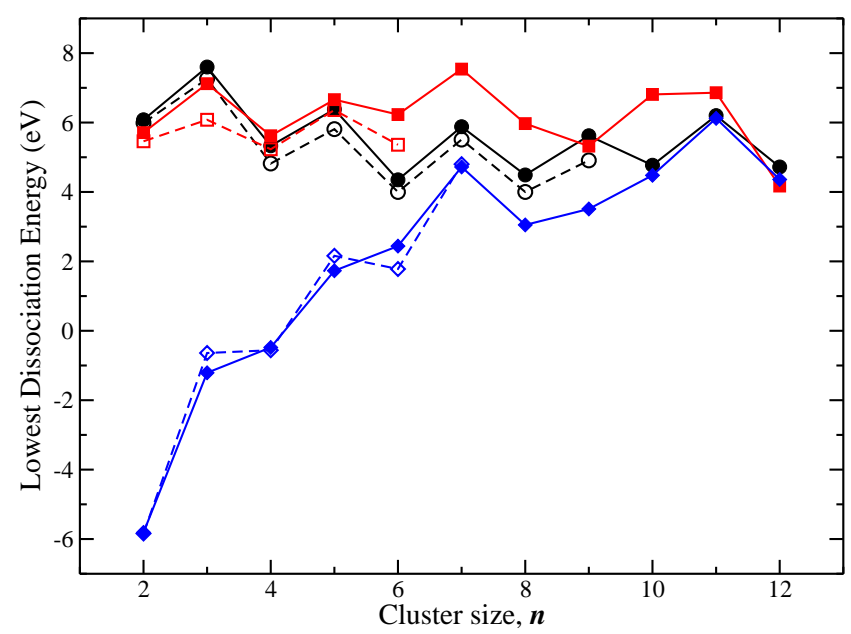

FIG. 5: Lowest dissociation energy (in eV) for the $\mathrm{C}_{n}^{q+}$ clusters. Circles: neutral clusters; squares: singly charged clusters; diamonds: doubly charged clusters; filled symbols and full lines: B3LYP results; open symbols and dashed lines: $\operatorname{CCSD}(\mathrm{T})$ results.

is shared by both fragments $\left(\mathrm{C}_{n}^{+} / \mathrm{C}_{n-k}^{+}\right)$present lower dissociation energy. In Fig. 4 alternations in the dissociation energy as a function of the cluster size are also shown, but in a lower degree as in neutral or cationic species.

A summary of the results obtained for dissociation energies is found in Fig. 5. In this figure we have plotted the lowest dissociation energy as a function of the cluster size at both levels of theory employed. One can see that differences between B3LYP and CCSD(T) predictions are rarely larger than $1 \mathrm{eV}$, being in most of the cases close to each other. Clusters with an odd number of carbon clusters are more stable: a larger dissociation energy is required to break these molecules. In particular, $\mathrm{C}_{3}$ presents the larger dissociation energy $(\sim 8 \mathrm{eV})$. For $n=9$ the dissociation of the cation becomes similar to that of the neutral, for $n>11$ these dissociation energies become similar.

\section{Fragmentation}

Experimental works focussed in fragmentation of neutral and charged small carbon clusters have been extensively carried out by Wohrer and coworkers (see e.g. [15-17, 19]). In these experiments they measure the branching ratios of all possible fragmentation channels. For example, the predominant fragmentation channel for $\mathrm{C}_{7}$ cluster is $\mathrm{C}_{4} / \mathrm{C}_{3}(47 \%)$, followed by $\mathrm{C}_{3} / \mathrm{C}_{3} / \mathrm{C}(14 \%), \mathrm{C}_{5} / \mathrm{C}_{2}(13 \%)$ and $\mathrm{C}_{3} / \mathrm{C}_{2} / \mathrm{C}_{2}(7 \%)$. Taking into account our results of dissociation energies we observe that the lowest dissociation energy channel corresponds to $\mathrm{C}_{4} / \mathrm{C}_{3}(5.51 \mathrm{eV})$, in agreement with the larger experimental branching ratio. However, in contrast with the experiment, channels $\mathrm{C}_{5} / \mathrm{C}_{2}(5.80 \mathrm{eV})$ and $\mathrm{C}_{6} / \mathrm{C}(6.33 \mathrm{eV})$ should present a larger branching ratio than the channel $\mathrm{C}_{3} / \mathrm{C}_{3} / \mathrm{C}$ $(10.33 \mathrm{eV})$. Thus, predictions solely based on energetic criteria are not sufficient to explain the measured branching ratios. For this reason we have applied the statistical method of mi- 


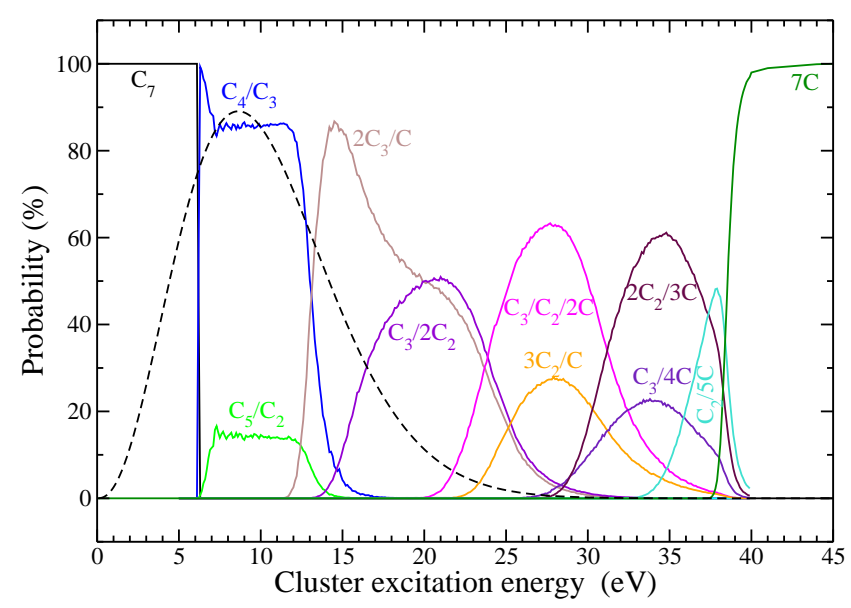

FIG. 6: Theoretical branching ratios as functions of the cluster excitation energy. Dashed line: cluster energy distribution multiplied by ten.

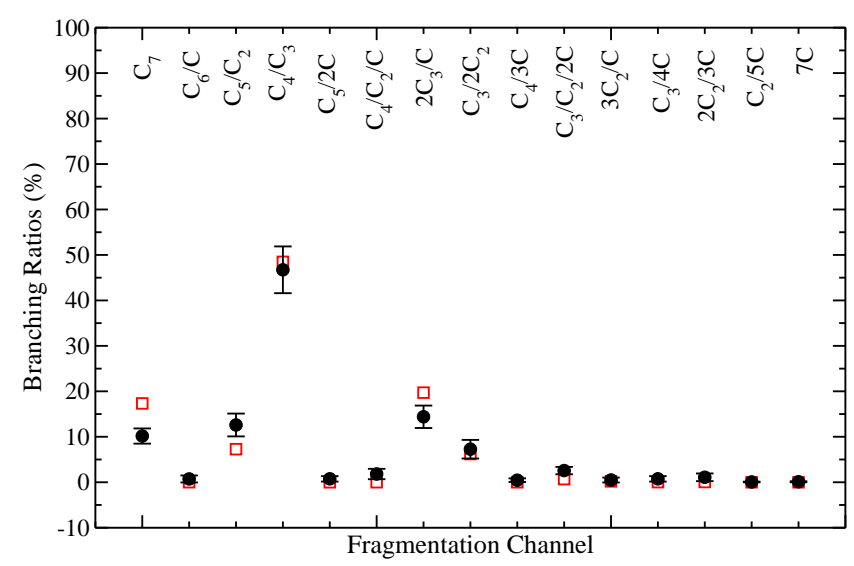

FIG. 7: Branching ratios for deexcitation of $\mathrm{C}_{7}$ cluster. Full circles: experiment; empty squares: convolution of the theoretical branching ratios with the energy distribution shown by a dashed line in Fig. 6 .

crocanonical Metropolis Monte Carlo (MMMC) presented in [22] to have a direct comparison with the experimental measurements.

As an example, Fig. 6 shows the theoretical branching ratios as functions of the cluster excitation energy obtained in a MMMC simulation of $\mathrm{C}_{7}$. The results show that the branching ratios present variations when the excitation energy is close to the dissociation thresholds. In addition, competition between different channels for a given excitation energy is also observed. The dominant channels are those presenting a $\mathrm{C}_{3}$ cluster. To compare the theoretical results with the experimental measurements one has to convolute the calculated branching ratios with the collisional energy deposit distribution. Following a similar procedure than the presented in ref. [19] we have obtained the energy distribution function shown in Fig. 6 (dashed line). Comparison of the theoretical results obtained with the experimental measurements is shown in Fig. 7. A good agreement is shown between our prediction and the experimental data. By far, the dominant channel is $\mathrm{C}_{4} / \mathrm{C}_{3}$. The rest of the channels have a BR below $20 \%$.

Thus, the presented procedure (combination of experimental branching ratios and MMMC simulations) allows us to extract the energy deposit just before the cluster fragmentation, i.e., in the collision process. This information is not available only from the theoretical point of view neither from the experimental one itself.

\section{CONCLUSIONS AND PERSPECTIVES}

We have presented a theoretical study of the ionization potentials, dissociation energies and fragmentation of neutral and positively charged small carbon clusters. In comparison with the more expensive CCSD(T) calculations, the DFT approach has been shown to be extremely useful in predicting accurate dissociation energies and ionization potentials. While first IP varies with the cluster size, showing a slow decrease, second IP is larger than the first one and decrease monotonically with $n$. Dissociation energies show alternation with the cluster size. As a general trend channels evaporating a cluster with an odd number of atoms (in particular $\mathrm{C}_{3}$ ) are more favorable. $\mathrm{C}_{5}^{2+}$ is the smallest doubly charged cluster which is thermodynamically stable against dissociation $\left(\mathrm{C}_{3}^{2+}\right.$ and $\mathrm{C}_{4}^{2+}$ are metastable). In the fragmentation dynamics we have observed competition between different dissociation channels for a given excitation energy. Comparison of our results with experimental measurements of $\mathrm{C}_{n}^{+}+\mathrm{He}$ collisions has allowed us to determine the energy distribution of the clusters just after the collision. Application of the MMMC method to charged species $\left(\mathrm{C}_{n}^{+}, n=5-8\right)$ and larger clusters $\left(\mathrm{C}_{60}\right.$ and $\left.\mathrm{C}_{60}^{+}\right)$will be also considered. For these systems dissociation energies and ionization potentials has been reported elsewhere [25].

\section{Acknowledgments}

We thank the Centro de Computación Científica de la Universidad Autónoma de Madrid (CCC-UAM) for allocation of computer time. Work partially supported by the DGI project Nos. BFM2003-00194, BQU2003-00894 and CTQ200400039/BQU, and the CAM project No. GR/MAT/0083/2004.
[1] P. P. Radi, M. E. Rincon, M. T. Hsu, J. Brodbelt-Lustig, P. R. Kemper, and M. T. Bowers, J. Chem. Phys. 92, 4817 (1990).

[2] P. P. Radi, G. V. Helden, M. T. Hsu, P. R. Kemper, and M. T. Bowers, Int. J. Mass Spectrom. Ion Proc. 109, 49 (1991).
[3] M. E. Geusic, T. J. McIlrath, M. F. Jarrold, L. A. Bloomfield, R. R. Freeman, and W. L. Brown, J. Chem. Phys. 84, 2421 (1986).

[4] M. E. Geusic, T. J. McIlrath, M. F. Jarrold, L. A. Bloomfield, 
R. R. Freeman, and W. L. Brown, Z. Phys. D 3, 309 (1986).

[5] M. E. Geusic, M. F. Jarrold, T. J. McIlrath, R. R. Freeman, and W. L. Brown, J. Chem. Phys. 86, 3862 (1987).

[6] R. Bouyer, F. R. Monchicourt, M. Perdix, and P. Pradel, J. Chem. Phys. 100, 8912 (1994).

[7] K. B. Shelimov, J. M. Hunter, and M. F. Jarrold, Int. J. Mass Spectrom. Ion Proc. 138, 17 (1994).

[8] M. B. Sowa, P. A. Hinz, and S. L. Anderson, J. Chem. Phys. 95, 4719 (1991).

[9] C. Lifshitz, T. Peres, S. Kababia, and I. Agranat, Int. J. Mass Spectrom. Ion Proc. 82, 193 (1988).

[10] C. Lifshitz, T. Peres, and I. Agranat, Int. J. Mass Spectrom. Ion Proc. 93, 149 (1989).

[11] C. Lifshitz, H. F. G. P. Sandler, J. Sun, T. Weiske, and H. Schwart, J. Phys. Chem. 97, 6592 (1993).

[12] S. W. McElvany, Int. J. Mass Spectrom. Ion Proc. 102, 81 (1990).

[13] Y. Tai, J. Murakami, Y. Maruyama, W. Yamaguchi, T. Mizota, K. Igarashi, and S. Tanemura, J. Phys. Chem. B 103, 5500 (1999).

[14] H. Choi, R. T. Bise, A. A. Hoops, D. H. Mordaunt, and D. M. Neumark, J. Phys. Chem. 104, 2025 (2000).

[15] M. Chabot, K. Wohrer, J. P. Rozet, D. Gardès, D. Vernhet, D. Jacquet, S. DellaNegra, A. Brunelle, M. Nectoux, M. Pautrat, et al., Phys. Scr. T73, 282 (1997).

[16] M. Chabot, R. Fossé, K. Wohrer, D. Gardés, G. Maynard, F. Ravilloud, and F. Spiegelman, Eur. Phys. J. D 14, 5 (2001).

[17] M. Chabot, S. D. Negra, L. Lavergne, G. Martinet, K. Wohrer, R. Sellem, R. Daniel, J. L. Bris, G. Lalu, D. Gardés, et al., Nucl. INucl. Instrum. Methods B 197, 155 (2002).

[18] G. Martinet, M. Chabot, K. Wohrer, S. D. Negra, D. Gardes, J. A. Scarpaci, P. Desesquelles, V. Lima, S. Díaz-Tendero, M. Alcamí, et al., Eur. Phys. J. D 24, 149 (2003).

[19] G. Martinet, S. Díaz-Tendero, M. Chabot, K. Wohrer, S. D. Negra, F. Mezdari, H. Hamrita, P. Désesquelles, A. L. Padellec, D. Gardés, et al., Phys. Rev. Lett. 93, 063401 (2004).

[20] W. Weltner and R. J. V. Zee, Chem. Rev. 89, 1713 (1989).

[21] A. V. Orden and R. J. Saykally, Chem. Rev. 98, 2313 (1998).

[22] S. Díaz-Tendero, P.-A. Hervieux, M. Alcamí, and F. Martín,
Phys. Rev. A 71, 033202 (2005).

[23] A. D. Becke, J. Chem. Phys. 98, 5648 (1993).

[24] C. Lee, W. Yang, and R. G. Parr, Phys. Rev. B 37, 785 (1988).

[25] S. Díaz-Tendero, M. Alcamí, and F. Martín, J. Chem. Phys. 119, 5545 (2003).

[26] J. M. L. Martin, J. El-Yazal, and J. P. François, Chem. Phys. Lett. 242, 570 (1995).

[27] J. M. L. Martin, J. El-Yazal, and J. P. François, Chem. Phys. Lett. 252, 9 (1996).

[28] S. Díaz-Tendero, F. Martín, and M. Alcamí, J. Phys. Chem. A 106, 10782 (2002).

[29] R. J. Barlett, Recent Advances in Coupled-Cluster Methods (World Scientific, Singapore, 1997).

[30] M. J. Frisch, G. W. Trucks, H. B. Schlegel, G. E. Scuseria, M. A. Robb, J. R. Cheeseman, V. G. Zakrzewski, J. A. M. Jr., R. E. Stratmann, J. C. Burant, et al., Gaussian 98, Revision A.11 (Gaussian, Inc., Pittsburgh PA, 2001).

[31] R. Ramanathan and J. A. Zimmerman, J. Chem. Phys. 98, 7838 (1993).

[32] J. Drowart, R. P. Burns, G. DeMaria, and M. G. Inghram, J. Chem. Phys. 31, 1131 (1959).

[33] F. J. Kohl and C. A. Stearns, J. Chem. Phys. 52, 6310 (1970).

[34] S. B. Bach and J. R. Eyler, J. Chem. Phys. 92, 358 (1990).

[35] C. Reid, J. A. Ballantine, S. R. Andrews, and F. M. Harris, Chem. Phys. 190, 113 (1995).

[36] K. P. Huber and G. Herzberg, Molecular Spectra and Molecular Structure, 4: Constants of Diatomic Molecules (Van Nostrand, New York, 1979).

[37] D. R. Lide, Handbook of chemistry and physics, 77th ed. (1996).

[38] R. L. Kelly, J. Phys. Chem. Ref. Data, Suppl. 91, 161 (1987).

[39] S. J. Blanksby, D. Schroder, S. Dua, J. H. Bowie, and H. Schwarz, J. Am. Chem. Soc. 122, 7105 (2000).

[40] M. G. Giuffreda, M. S. Deleuze, and J. P. François, J. Phys. Chem. A 103, 5137 (1999).

[41] H. Hogreve, J. Chem. Phys. 102, 3281 (1995).

[42] H. Hogreve, J. Mol. Struct. (THEOCHEM) 532, 81 (2000).

[43] H. Hogreve and A. F. Jalbout, J. Chem. Phys. 119, 8849 (2003). 